\title{
Dissociation of Pharmacokinetic-Pharmacodynamic Success and Clinical Failure of Tazobactam/Piperacillin in Escherichia coli Bacteremia: A Case Report
}

\author{
Hiroki Magarifuchi ${ }^{\mathrm{a}, \mathrm{b}, \mathrm{c}}$, Toshiharu Urakami ${ }^{\mathrm{a}, \mathrm{b}}$, Hiroki Yamakuchi ${ }^{\mathrm{a}}$, \\ Yohei Hamada ${ }^{\text {a }}$, Yosuke Aoki ${ }^{\mathrm{a}}$
}

\begin{abstract}
An 84-year-old woman presenting with fever and the right lower guardant pain was found to have ischemic colitis and left renal abscess on CT scan findings, and started on empiric antimicrobial therapy with intravenous tazobactam/piperacillin (TAZ/PIPC) 4.5 g q8h. The blood culture prior to TAZ/PIPC grew Escherichia coli (E. coli) with MIC of $2 \mathrm{~g} / \mathrm{mL}$. The patient's fever still continued; however, the follow-up blood cultures on another three occasions all demonstrated intermittent $E$. coli bacteremia despite continuation of TAZ/PIPC (MIC ranging from $\leq 2$ to $16 \mathrm{~g} / \mathrm{mL}$ ). To make sure proper distribution of the drug, the pharmacokinetic parameters were quantitated after 18 dosing based on the serum concentration of TAZ/PIPC applied to the Sawchuk-Zaske equation: the serum peak/trough levels for PIPC and TAZ were $269 / 11 \mathrm{~g} / \mathrm{mL}$ and $41 / 5 \mathrm{~g} / \mathrm{mL}$, with the time above MIC (T > MIC) of PIPC ranging from $70 \%$ to $100 \%$. The volume of distribution of PIPC was 13.82 $\mathrm{L}$, total clearance $98.89 \mathrm{~mL} / \mathrm{min}$, elimination rate constant (kel) $0.43 \mathrm{~h}^{-1}$, and resultant plasma half-life $(0.693 / \mathrm{kel}) 1.6 \mathrm{~h}$. Although $\mathrm{T}>\mathrm{MIC}$ thus reflected optimal pharmacokinetics of TAZ/PIPC against Gram-negative blood stream infection, since blood cultures remained positive, the antimicrobial regimen was switched to intravenous pazufloxacin and tobramycin. The altered therapeutic regimen resulted in sterilization of the blood culture and gradual disappearance of the renal abscess. To the best of our literature search, this case report is the first to demonstrate on the basis of patient's pharmacokinetic profile that the maximal attainment of pharmacological target of beta-lactam ( $\mathrm{T}>\mathrm{MIC}$ ) does not always provide reassurance of successful treatment of blood stream infections.
\end{abstract}

Keywords: Escherichia coli; Refractory bacteremia; Renal abscess; Tazobactam/piperacillin; Time above MIC

Manuscript accepted for publication September 11, 2013

${ }^{a}$ Division of Infectious Disease and Hospital Epidemiology, Saga University Hospital, Saga, Japan

${ }^{\mathrm{b}}$ These two authors equally contributed to this scientific case report.

${ }^{\mathrm{c} C}$ Corresponding author: Hiroki Magarifuchi, 5-1-1, Nabeshima, Saga

849-8501, Japan. Email: f8817@cc.saga-u.ac.jp

doi: http://dx.doi.org/10.4021/jmc1494w

\section{Introduction}

Clinical efficacy of beta-lactam antibiotics shows close correlation with a pharmacokinetic-pharmacodynamic (PK-PD) parameter, time above MIC ( $\mathrm{T}>\mathrm{MIC})$, during which the serum concentration of a given drug exceeds its MIC (T > MIC) against the causative pathogen [1]. As with penicillins, $\mathrm{T}>\mathrm{MIC}$ of $30 \%$ and $50 \%$, correlates well with its bacteriostatic, and bactericidal effect [2].

Tazobactam/piperacillin (TAZ/PIPC) is an anti-pseudomonal broad spectrum penicillin combined with a beta-lactamase inhibitor, tazobactam (TAZ) at a ratio of 1 (TAZ):8 (PIPC). The high clinical efficacy of this drug in patients with sepsis has been confirmed in the phase III trials in Japan, including bacteremia caused by beta-lactamase producing Gram-negative rods [3]. Recent report suggests that TAZ/ PIPC would potentially be a good choice even in the bacteremia due to ESBL (extended-spectrum beta-lactamase) producers given that the causative strains are sensitive in vitro to this drug [4]. Thus, TAZ/PIPC has shown a lot of clinical efficacy in the era of serious infections due to antimicrobial resistant pathogens.

We report on a case of $E$. coli bacteremia associated with renal abscess, in whom bacteremia was refractory to TAZ/ PIPC treatment even though the time above MIC persistently exceeded $70 \%$. The pathophysiology of the refractory bacteremia is discussed in light of PK-PD analyses with review of the pertinent literatures.

\section{Case Report}

An 84-year-old woman was referred to Saga University Hospital for work-up of fever, and the right lower guardant pain associated with diarrhea. Her past medical history included hypertension and reflux esophagitis.

On admission, the body temperature was $38{ }^{\circ} \mathrm{C}$, pulse rate $112 / \mathrm{min}$, respiratory rate $24 / \mathrm{min}$, and blood pressure $99 / 66 \mathrm{mmHg}$. The oxygen saturation was $95 \%$ while the patient was breathing $2 \mathrm{~L} / \mathrm{min}$ oxygen. The abdomen was soft with tenderness in the right lower quadrant area. The labo- 
\begin{tabular}{l|l}
\hline TAZ/PIPC & TOB \\
\hline
\end{tabular}

\begin{tabular}{|l|l|}
\hline PZFX & CPFX \\
\hline
\end{tabular}

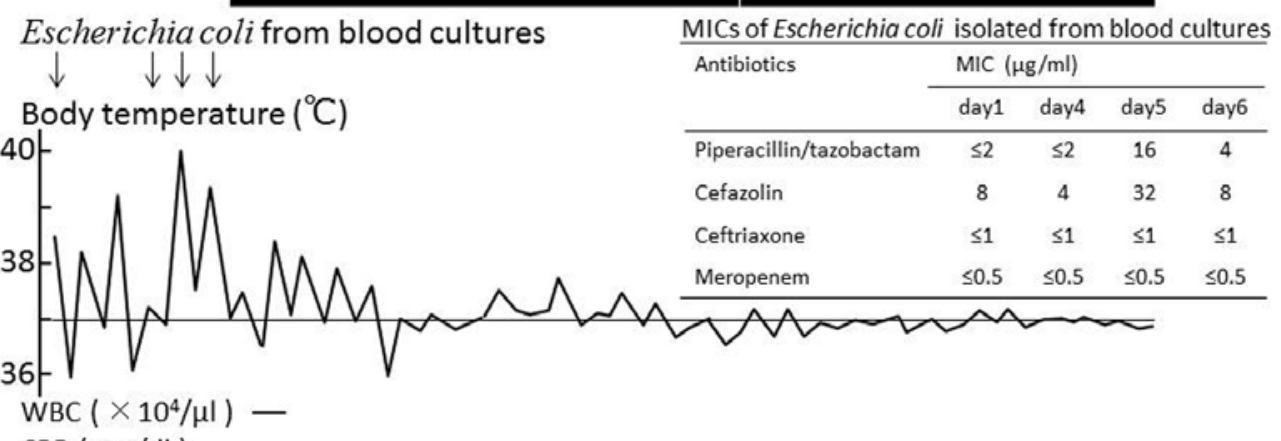

CRP ( $\mathrm{mg} / \mathrm{dl}) \cdots$

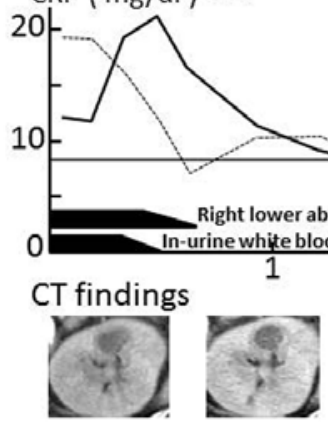

Figure 1. Clinical course. Right lower abdominal pain improved and pyuria turned negative after administering TAZ/PIPC, but fever and bacteremia still continued. After TAZ/PIPC was replaced with PZFX and TOB, fever subsided and blood culture was sterilized. Abdominal CT (bottom) shows gradual reduction in size and subsequent disappearance of the left renal abscess after antimicrobial switch to PZFX. TAZ/PIPC: Tazobactam/piperacillin $4.5 \mathrm{~g} \times 3 \mathrm{div}$; TOB: Tobramycin $150 \mathrm{mg} \times 1 \mathrm{div}$; PZFX: Pazufloxacin 500 mg × 1-2 div; CPFX: Ciprofloxacin 400 mg/day p.o. MICs were determined by microdilution method.

ratory results on admission showed peripheral WBC count $15,800 / \mathrm{L}$ with significant increase in polymorphonuclear leukocytes, the serum level of C-reactive protein $19.6 \mathrm{mg} /$ $\mathrm{dL}$, and the blood gas analysis revealed metabolic acidosis with respiratory compensation. These clinical features suggested severe sepsis with septic shock. The urinalysis was positive for leukocytes and nitrate, but the Gram stain was negative for bacteria. The plain abdominal X-ray found distension of the ascending colon, which was revealed by contrast-enhanced CT scan to have been caused by ischemic colitis.

There was also a low density lesion with peripheral enhancement in the left kidney that was suggestive of abscess in the corticomedullary portion (Fig. 1).

After drawing two sets of blood culture, empiric treatment of intravenous TAZ/PIPC $4.5 \mathrm{~g} \mathrm{q} 8 \mathrm{H}$, adjusted to creatinine clearance $(67 \mathrm{~mL} / \mathrm{min})$, was started. The blood culture subsequently grew $E$. coli with the MIC of ampicillin $(\mathrm{ABPC})>32 \mathrm{~g} / \mathrm{mL}$, sulbactam/ampicillin (SBT/ABPC) 32 $\mathrm{g} / \mathrm{mL}$, PIPC $>64 \mathrm{~g} / \mathrm{mL}$, and TAZ/PIPC $\leq 2 \mathrm{~g} / \mathrm{mL}$. The right lower quadrant pain and pyuria showed improvements in response to TAZ/PIPC. The fever was still present; however, and the repeat blood culture drawn on the 4th, 5th, and 6th hospital-day all retrieved $E$. coli despite continuing antimicrobial treatment. The MICs of TAZ/PIPC against all the strains of $E$. coli ranged from 2 to $16 \mathrm{~g} / \mathrm{mL}$ (Fig. 1), which was considered sensitive.

The possible cause of the breakthrough bacteremia observed at this point was deemed either persistence of renal abscess from which $E$. coli spread into blood stream, or altered distribution of TAZ/PIPC, such as declining of serum concentration due to septic shock-induced increase in capillary permeability.

PK parameters were individually derived by measuring the patient's serum concentrations of TAZ and PIPC separately, with the use of HPLC and the Sawchuk-Zaske equation as described previously [7, 8]. Subsequently the patient specific profile of the concentration time curve was simulated, and T > MIC was calculated by using one-compartmental single-dose infusion model. The serum peak/trough levels were $269 / 11 \mathrm{~g} / \mathrm{mL}$ for PIPC and $41 / 5 \mathrm{~g} / \mathrm{mL}$ for TAZ. The percent of time during which the serum concentration of TAZ/PIPC exceeds the MICs of the E. coli strains ranged from $70 \%$ at the MIC of $16 \mathrm{~g} / \mathrm{mL}$ to $100 \%$ at the MIC of $2 \mathrm{~g} /$ 


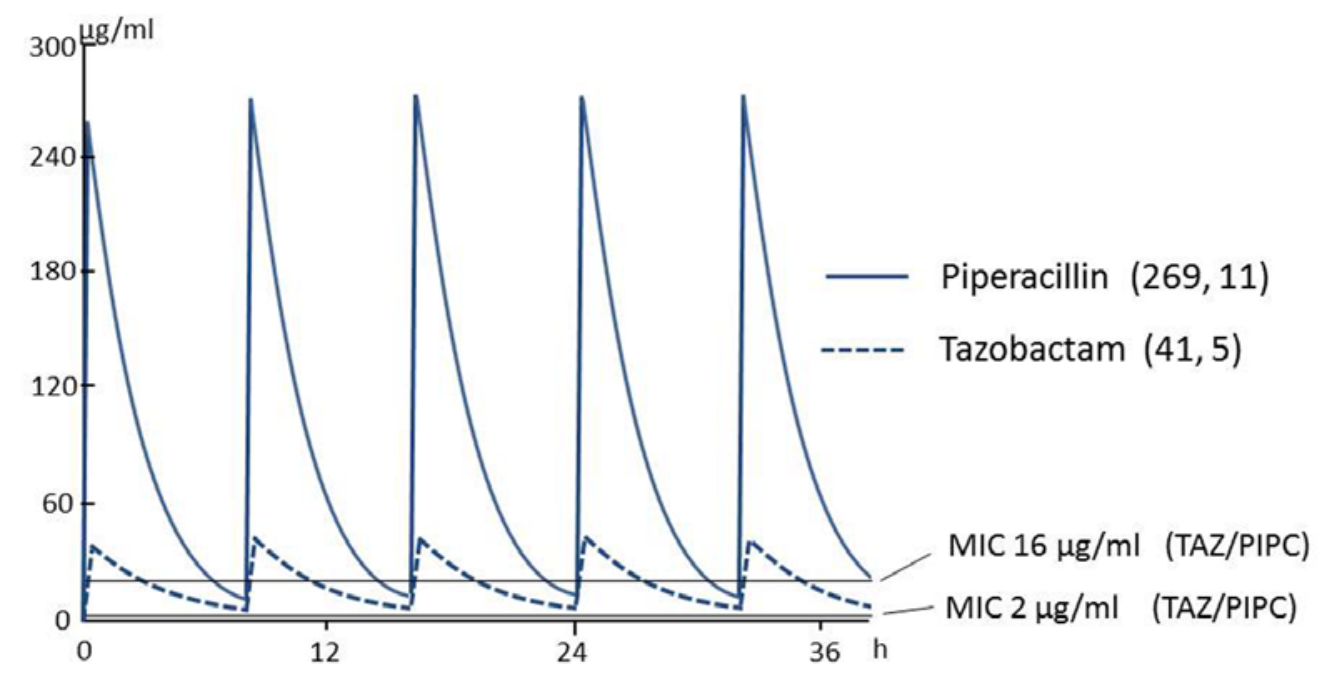

The case profile: Age; 84, Sex; woman, Body weight; 48 kg, Estimated creatinine clearance; 67 $\mathrm{ml} / \mathrm{min}$, Antimicrobial dose; TAZ/PIPC $4.5 \mathrm{~g} \times 3$ (After 18 doses), Pathogen; Escherichia coli (MIC 2-16)

Figure 2. Pharmacokinetics-pharmacodynamics analysis. The serum peak/trough levels were $269 / 11 \mu \mathrm{g} / \mathrm{mL}$ for PIPC and $41 / 5 \mu \mathrm{g} / \mathrm{mL}$ for TAZ. The time during which the serum concentration of TAZ/PIPC exceeds the MICs of the E. coli strains ranged from $70 \%$ at the MIC of $16 \mu \mathrm{g} / \mathrm{mL}$ to $100 \%$ at the MIC of $2 \mu \mathrm{g} / \mathrm{mL}$. Serum concentrations of piperacillin and tazobactam were quantitated by HPLC, and pharmacokinetic parameters were determined using Sawchuk-Zaske equation as described previously $[5,6]$.

$\mathrm{mL}$ (Fig. 2). The volume of distribution (VD), the total clearance of the drug $\left(\mathrm{CL}_{\mathrm{T}}\right)$, and the elimination half-life $\left(\mathrm{T}_{1 / 2}\right)$ was $13.82 \mathrm{~L}$ (reference range: $9.56 \pm 6.59$ [7], $11.9 \pm 1.70$ [8], $11.6 \pm 2.1[9]), 98.89 \mathrm{~mL} / \mathrm{min}$ (reference range: 173.5 $\pm 75.33[7], 181.67 \pm 20.00[8], 183 \pm 41[9])$, and $1.61 \mathrm{~h}$ (reference range: $0.844 \pm 0.083$ [9]), respectively (Table 1).

Although the drug distribution of TAZ/PIPC, as represented by CLT and T1/2, was thus altered to some extent, the high percentage of $\mathrm{T}>$ MIC made it unlikely that the breakthrough bacteremia was due to insufficient dosing of TAZ/PIPC (Fig. 2).

The follow-up CT scan revealed improvements of inflammatory findings of the ascending colon, but the size of the renal abscess remained the same as the pretreatment level (Fig. 1). Having confirmed clinical ineffectiveness despite the optimal in vivo pharmacokinetics of TAZ/PIPC, the antibiotic regimen was switched to pazufloxacin (PZFX) 500 $\mathrm{mg} \mathrm{q12h}$ and tobramycin (TOB) $180 \mathrm{mg} \mathrm{q} 24 \mathrm{~h}$ aiming at both

Table 1. Pharmacokinetics of Piperacillin/Tazobactam

\begin{tabular}{llllll}
\hline & VD $(\mathbf{L})$ & $\mathbf{C L}(\mathbf{m L} / \mathbf{m i n})$ & $\mathbf{C}_{\max }(\mathbf{m g} / \mathbf{m L})$ & $\mathbf{k e l}^{\left(\mathbf{h}^{-1}\right)}$ & $\mathbf{T}_{\mathbf{1} / 2}(\mathbf{h})$ \\
\hline Present case & 13.82 & 98.89 & 269 & 0.43 & 1.61 \\
Koomanachai et al [7] & $9.56 \pm 6.59$ & $173.5 \pm 75.33$ & $\mathrm{NA}$ & 0.92 & 0.75 \\
Kuti et al [8] & $11.9 \pm 1.70$ & $181.67 \pm 20.00$ & NA & 1.09 & 0.64 \\
Shiba [9] & $11.6 \pm 2.1$ & $183 \pm 41$ & 316 & 0.821 & 0.844 \\
\hline
\end{tabular}

VD: volume of distribution; CL: total drug clearance; kel: elimination rate constant; $\mathrm{T}_{1 / 2}$ : elimination half-time; NA: not available. 
adequate tissue penetration and eradication of bacteria from the blood stream. The fever gradually subsided following antibiotic therapy of PZFX + TOB, and the blood culture turned negative in parallel with disappearance of the renal abscess (Fig. 1). The patient's clinical course was uneventful thereafter, and was discharged to home.

\section{Discussion}

The clinical cues in this case were positive blood culture of $E$. coli, the right lower quadrant pain attributable to ischemic colitis of the ascending colon, and abscess formation in corticomedullary region of the left kidney without bacteriuria. These clinical features indicate in concert that the patient had developed translocation bacteremia of $E$. coli from ischemic colitis, which also hematogenously developed renal cortical abscess.

Despite high-dose intravenous administration of TAZ/ PIPC, E. coli bacteremia in our case was refractory to this antimicrobial therapy even though the PK-PD analyses confirmed the T $>$ MIC of TAZ/PIPC being kept mostly at $100 \%$ during the treatment. Penicillin antibiotics are known to exert bacteriostatic effects with $\mathrm{T}>$ MIC greater than $30 \%$, which is further enhanced to bactericidal when achieving greater than 50\% [2]. In Gram-negative infections, however, these values are expected to exceed $40 \%$ and $70 \%$ because of the absence of post-antibiotic effects against Gram-negative bacilli [10]. The same PK-PD profile can be applied to the TAZ/PIPC administered in the present case, because TAZ, a potent inhibitor of most group 2 beta-lactamase is known not to affect the pharmacokinetics of PIPC [11]. Since the infusion method (continuous infusion over $24 \mathrm{~h}$ vs. intermittent infusion) reportedly had no influence on $\mathrm{PK}$ parameters of TAZ/PIPC [12], our antimicrobial strategies of switching to other agents, not changing the dosing regimen, could be justified.

It is well known that beta-lactam agent is generally incapable of eradicating bacteria from circumscribed collection of purulent exudate such as abscess as seen in our case. This results from less effective tissue penetration of beta-lactams than other classes of antibiotics with higher VD, or lipophilic agents such as macrolide or fluoroquinolone. Therefore, given that intermittent bacteremia, which is discontinuous seeding of the same pathogen, is caused by infections such as abscesses [13], failure of TAZ/PIPC in sterilizing renal abscess may well explain refractory nature of $E$. coli bacteremia. But, why did it also fail in eradicating bacteria in the blood flow? The clinical picture with the use of TAZ/PIPC could have been persistent renal abscess with sterilization of blood culture, which was not observed in this patient.

In an acidic environment such as abscess, the MIC50/90 of TAZ/PIPC against 59 clinical isolates of Bacteroides fragilis was reportedly increased from $4 / 8 \mathrm{~g} / \mathrm{mL}(\mathrm{pH} 7.1)$ to $4 / 16 \mathrm{~g} / \mathrm{mL}(\mathrm{pH} 6.3$ ), then to $16 / 32 \mathrm{~g} / \mathrm{mL}(\mathrm{pH} \mathrm{5.8)} \mathrm{[14].}$ These findings may indicate that acidic $\mathrm{pH}$ confers bacteria a temporally enhanced antimicrobial resistance beyond the value of antimicrobial susceptibility as measured by MIC. Additionally, duration of the contact time of antibiotics at sufficient concentration and bacteria, which should be long enough to exert antimicrobial activity [15], might have been short enough with $\mathrm{q} 8 \mathrm{~h}$ dosing of TAZ/PIPC for bacteria to evade bactericidal effect of TAZ/PIPC.

In conclusion, we presented a case demonstrating $\mathrm{PK}$ PD success resulting in clinical failure of bacteremia. Infectious disease practitioner should be reminded that PK-PD theory should be carefully applied to different patients with different underlying conditions.

\section{Conflict of Interests}

None.

\section{References}

1. Craig WA. Pharmacokinetic/pharmacodynamic parameters: rationale for antibacterial dosing of mice and men. Clin Infect Dis. 1998;26(1):1-10; quiz 11-12.

2. Drusano GL. Prevention of resistance: a goal for dose selection for antimicrobial agents. Clin Infect Dis. 2003;36(Suppl 1):S42-50.

3. Shiba K, Ishihara S, Kawai S, Mikamo H, Yokoyama T. Phase III tazobactam/ piperacillin (1:8) study in patients with sepsis or infective endocarditis. Jpn J Chemother. 2010;58(S-1):73-87.

4. Rodriguez-Bano J, Navarro MD, Retamar P, Picon E, Pascual A. beta-Lactam/beta-lactam inhibitor combinations for the treatment of bacteremia due to extendedspectrum beta-lactamase-producing Escherichia coli: a post hoc analysis of prospective cohorts. Clin Infect Dis. 2012;54(2):167-174.

5. Urakami T, Aoki Y, Fukuoka M, Magarifuchi H, Nagata M, Nagasawa Z, Nakano Y, et al. Application of Betalactam Therapeutic Drug Monitoring in Clinical Practice Using HPLC. J Bioanal Biomed. 2011;3:6.

6. Aoki Y, Urakami T, Magarifuchi H, Nagasawa Z, Nagata M, Fukuoka M. The importance of pharmacokinetic consultation of cefepime treatment for Pseudomonas aeruginosa bacteremia: a case report of severe thermal burn injury. J Infect Chemother. 2011;17(3):407-411.

7. Koomanachai P, Bulik CC, Kuti JL, Nicolau DP. Pharmacodynamic modeling of intravenous antibiotics against gram-negative bacteria collected in the United States. Clin Ther. 2010;32(4):766-779.

8. Kuti JL, Nightingale CH, Nicolau DP. Optimizing phar- 
macodynamic target attainment using the MYSTIC antibiogram: data collected in North America in 2002. Antimicrob Agents Chemother. 2004;48(7):2464-2470.

9. Shiba K. Phase I study of tazobactam/piperacillin in healthy volunteers. Jpn J Chemother. 2010;58(S-1):110.

10. Craig WA. The role of pharmacodynamics in effective treatment of community-acquired pathogens. Adv Stud Med. 2002;2:126-134.

11. Bush LM, Johnson CC. Ureidopenicillins and beta-lactam/beta-lactamase inhibitor combinations. Infect Dis Clin North Am. 2000;14(2):409-433, ix.

12. Li C, Kuti JL, Nightingale CH, Mansfield DL, Dana A, Nicolau DP. Population pharmacokinetics and pharma- codynamics of piperacillin/tazobactam in patients with complicated intra-abdominal infection. J Antimicrob Chemother. 2005;56(2):388-395.

13. Seifert H. The clinical importance of microbiological findings in the diagnosis and management of bloodstream infections. Clin Infect Dis. 2009;48(Suppl 4):S238-245.

14. Falagas ME, McDermott L, Snydman DR. Effect of $\mathrm{pH}$ on in vitro antimicrobial susceptibility of the Bacteroides fragilis group. Antimicrob Agents Chemother. 1997;41(9):2047-2049.

15. Hessen MT, Kaye D. Principles of selection and use of antibacterial agents. In vitro activity and pharmacology. Infect Dis Clin North Am. 2000;14(2):265-279, vii. 